\title{
Correction to: Phenolic Profiling of Medicinal Species of Chuquiraga, Asteraceae, by HPLC Fingerprinting
}

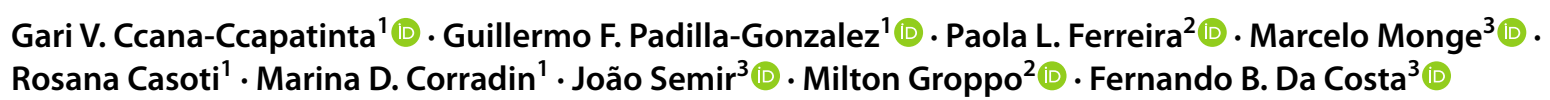

Published online: 17 November 2021

(c) The Author(s) under exclusive licence to Sociedade Brasileira de Farmacognosia 2021

Correction to: Revista Brasileira de Farmacognosia https://doi.org/10.1007/s43450-021-00177-y

This article has been updated.

The original article can be found online at https://doi.org/10.1007/ s43450-021-00177-y.

Fernando B. Da Costa febcosta@fcfrp.usp.br

1 Laboratório de Farmacognosia, Faculdade de Ciências Farmacêuticas de Ribeirão Preto, Universidade de São Paulo, Ribeirão Preto, São Paulo, Brazil

2 Departamento de Biologia, Faculdade de Filosofia, Ciências E Letras de Ribeirão Preto, Universidade de São Paulo, Ribeirão Preto, São Paulo, Brazil

3 Instituto de Biologia, Universidade de Campinas, Campinas, São Paulo, Brazil 